\title{
Radial Spin Texture of the Weyl Fermions in Chiral Tellurium
}

\author{
G. Gatti®${ }^{1,2}$ D. Gosálbez-Martínez, ${ }^{1,3}$ S. S. Tsirkin $\odot,{ }^{4}$ M. Fanciulliø ${ }^{5,6}$ M. Puppin $\odot,{ }^{7,2}$ S. Polishchuk $\odot,{ }^{7,2}$ S. Moser $\odot,{ }^{8,9}$ \\ L. Testa $\odot,{ }^{1}$ E. Martino $\odot,{ }^{1}$ S. Roth, ${ }^{1,2}$ Ph. Bugnon, ${ }^{1}$ L. Moreschini $\odot,{ }^{8}$ A. Bostwick, ${ }^{8}$ C. Jozwiak $\odot,{ }^{8}$ E. Rotenberg $\odot,{ }^{8}$ G. Di \\ Santo $\odot,{ }^{10}$ L. Petaccia $\odot,{ }^{10}$ I. Vobornik, ${ }^{11}$ J. Fujii, ${ }^{11}$ J. Wong $\odot,{ }^{12}$ D. Jariwala $\odot,{ }^{13}$ H. A. Atwater, ${ }^{12}$ H. M. Rønnow $\odot,{ }^{1}$ \\ M. Chergui $\odot^{7,2}$ O. V. Yazyev $\odot{ }^{1,3}$ M. Grioni $\oplus^{1,2}$ and A. Crepaldi $\oplus^{1,2, *}$ \\ ${ }^{1}$ Institute of Physics, Ecole Polytechnique Fédérale de Lausanne (EPFL), CH-1015 Lausanne, Switzerland \\ ${ }^{2}$ Lausanne Centre for Ultrafast Science (LACUS), Ecole Polytechnique Fédérale de Lausanne (EPFL), \\ CH-1015 Lausanne, Switzerland \\ ${ }^{3}$ National Centre for Computational Design and Discovery of Novel Materials MARVEL, \\ Ecole Polytechnique Fédérale de Lausanne (EPFL), CH-1015 Lausanne, Switzerland \\ ${ }^{4}$ Department of Physics, University of Zurich, Winterthurerstrasse 190, 8057 Zurich, Switzerland \\ ${ }^{5}$ Laboratoire de Physique des Matériaux et Surfaces, CY Cergy Paris Université, 95031 Cergy-Pontoise, France \\ ${ }^{6}$ Université Paris-Saclay, CEA, CNRS, LIDYL, 91191 Gif-sur-Yvette, France \\ ${ }^{7}$ Laboratory of Ultrafast Spectroscopy, ISIC, Ecole Polytechnique Fédérale de Lausanne (EPFL), \\ CH-1015 Lausanne, Switzerland \\ ${ }^{8}$ Advanced Light Source, Lawrence Berkeley National Laboratory, Berkeley, California 94720, USA \\ ${ }^{9}$ Physikalisches Institut and Würzburg-Dresden Cluster of Excellence ct.qmat, Universität Würzburg, \\ 97074 Würzburg, Germany \\ ${ }^{10}$ Elettra Sincrotrone Trieste, Strada Statale $14 \mathrm{~km}$ 163.5, 34149 Trieste, Italy \\ ${ }^{11}$ CNR-IOM, TASC Laboratory, Area Science Park-Basovizza, 34139 Trieste, Italy \\ ${ }^{12}$ Department of Applied Physics, California Institute of Technology, Pasadena, California 91125, USA \\ ${ }^{13}$ Department of Electrical and Systems Engineering, University of Pennsylvania, Philadelphia, Pennsylvania 19104, USA
}

(Received 22 July 2020; revised 15 September 2020; accepted 2 October 2020; published 19 November 2020)

\begin{abstract}
Trigonal tellurium, a small-gap semiconductor with pronounced magneto-electric and magneto-optical responses, is among the simplest realizations of a chiral crystal. We have studied by spin- and angleresolved photoelectron spectroscopy its unconventional electronic structure and unique spin texture. We identify Kramers-Weyl, composite, and accordionlike Weyl fermions, so far only predicted by theory, and show that the spin polarization is parallel to the wave vector along the lines in $k$ space connecting high-symmetry points. Our results clarify the symmetries that enforce such spin texture in a chiral crystal, thus bringing new insight in the formation of a spin vectorial field more complex than the previously proposed hedgehog configuration. Our findings thus pave the way to a classification scheme for these exotic spin textures and their search in chiral crystals.
\end{abstract}

DOI: 10.1103/PhysRevLett.125.216402

Since the first synthesis of single crystals [1], the study of $\mathrm{Te}$ has continuously disclosed novel phenomena. The magneto-electric effect is the hallmark of $\mathrm{Te}$, the investigation of which dates back to the pioneering report of the galvanomagnetic effects by von Klitzing [2,3] and photogalvanic measurements by Asnin et al. [4,5]. Tellurium exhibits magneto-electric chiral anisotropy [6], current-induced bulk magnetization [7], and a kinetic Faraday effect $[8,9]$, and it thus realizes the equivalent of a quantum solenoid $[10,11]$. Theory predicts a current-

Published by the American Physical Society under the terms of the Creative Commons Attribution 4.0 International license. Further distribution of this work must maintain attribution to the author(s) and the published article's title, journal citation, and DOI. induced anomalous Hall effect [12] and quantized circular photogalvanic effect [13], which are related to kinetic magneto-electric and kinetic Faraday effects $[12,14]$. The large thermopower $[15,16]$ and the possibility of realizing field effect transistors [17] from exfoliated 2D flakes suggest promising practical applications.

Te has gained attention in the field of topological materials with the prediction [18-22], only partially confirmed by experiments [23], that it contains exotic Weyl fermions. It has been suggested as a platform to control topological phase transitions between a Weyl semiconductor, a Weyl semimetal, and a topological insulator [24], and signatures of a pressure-induced topological transition have recently been reported by magneto-transport [25] and optical studies [26]. The unique response of Te to external magnetic fields and the presence of Weyl fermions are related to the large Berry curvature combined with the 


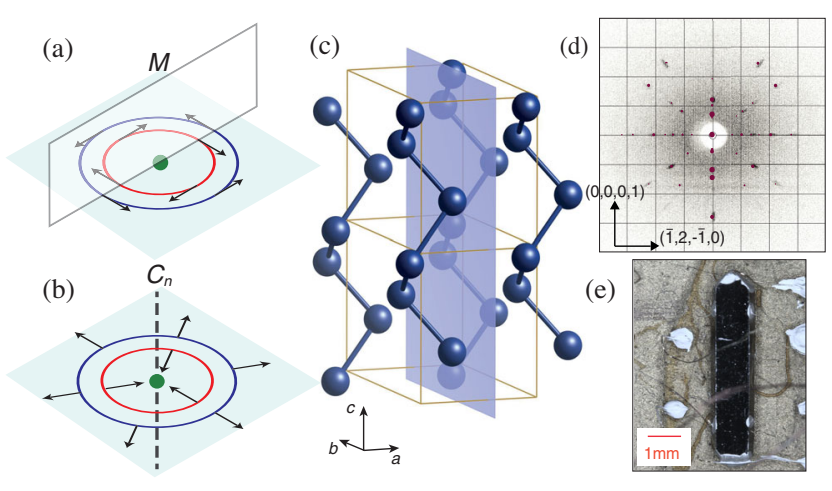

FIG. 1. Crystal structure and symmetries. (a) Sketch of the helical spin texture in momentum space for a Rashba system; due to the mirror symmetry $M$, the spin vector circulates around the high-symmetry point (green dot). (b) Radial spin texture in a chiral crystal; the screw rotational axis $C_{n}$ imposes a radial configuration in a plane passing through a high-symmetry point. (c) Crystal structure of trigonal tellurium, with the cleavage plane indicated in blue. (d) Laue diffraction pattern of a Te crystal investigated by angle-resolved photoemission spectroscopy (ARPES). The surface termination corresponds to the (1010) plane. (e) Single crystal of Te of several mm size.

spin polarization of the electronic states [12]. Therefore, a detailed investigation of its spin properties is timely.

In this Letter, we discuss the unconventional spin polarization of the Weyl fermions, which we measured by means of spin- and angle-resolved photoelectron spectroscopy. The exotic alignment of the spins around the Weyl points is a consequence of the chiral symmetry and a fundamental ingredient of the magneto-electric effect [10]. In crystals lacking inversion symmetry $(P)$, Kramers' spin degeneracy only holds at the time-reversal invariant momenta (TRIM) of the Brillouin zone (BZ) owing to the action of time-reversal symmetry $(T)$. The additional presence of a mirror symmetry $(M)$ forces the spin vector to be locked orthogonally to the electron wave vector. The resulting helical spin texture [Fig. 1(a)] is commonly observed at the surface of metals [27] and of topological insulators [28] and in the bulk of noncentrosymmetric materials [29]. By contrast, in a chiral crystal like Te, where both $P$ and $M$ are absent, the spin acquires a radial structure, illustrated in a 2D representation in Fig. 1(b). By comparing measurements at different high-symmetry points, we clarify the symmetry conditions that enforce a unique spin arrangement, not only at the Fermi surface, as recently observed by Sakano et al. [30], but also for symmetry protected Kramers-Weyl fermions.

Crystals of elemental Te belong to the trigonal system. Within point group 32, two enantiomeric structures are possible, with space groups $\mathrm{P} 3_{1} 21$ (152, right-handed) and $\mathrm{P}_{2} 21$ (154, left-handed), respectively. Figure 1(c) displays the unit cell of the right-handed enantiomer with lattice parameters $a=b=4.44 \AA$ and $c=5.91 \AA$ [31]. Covalently bonded helical chains run along the crystallographic $c$ axis, and the unit cell contains three atoms. The interaction between the chains is of the van der Waals type, and the crystal cleaves easily along the (1010) surface, as confirmed by x-ray Laue diffraction [Fig. 1(d)] and in good agreement with a previous low-energy electron diffraction study [32]. The $C_{3_{1}}$ screw axis runs along the $c$ direction, and three additional $C_{2}$ rotational axes lie in the plane orthogonal to $c$ [33].

High-quality single crystals postcleaved in ultrahigh vacuum exposed large mirrorlike surfaces [see Fig. 1(e)] suitable for ARPES studies. We determined the periodicity of the electronic structure along the $k_{z}$ direction orthogonal to the surface at the MAESTRO beamline 7.0.2 of the Advanced Light Source by varying the photon energy between 60 and $160 \mathrm{eV}$, with energy and angular resolutions equal to $20 \mathrm{meV}$ and $0.3^{\circ}$, respectively. We exploited high-resolution ARPES combined with alkali metal $(\mathrm{K})$ deposition to probe the band gap and the conduction band at the BaDElPh beamline [34] of the Elettra synchrotron, with $19 \mathrm{eV}$ photon energy and resolutions set to $10 \mathrm{meV}$ and $0.2^{\circ}$, respectively. Finally, the vectorial spin polarization has been mapped at the Elettra APE beamline [35]. The energy and angular resolution of the spin- and angle-resolved photoelectron spectroscopy were $50 \mathrm{meV}$ and $0.75^{\circ}$. In all experiments the sample temperature was set to $80 \mathrm{~K}$.

We calculated the electronic structure of trigonal $\mathrm{Te}$ within density functional theory using plane wave and fully relativistic norm-conserving pseudopotentials, as implemented in the QUANTUM ESPRESSO package [36]. The energy cut of the plane wave was set to $80 \mathrm{Ry}$, and we used a $8 \times 8 \times 6$ Monkhorst-Pack grid. The exchange and correlation interactions were treated within the PerdewBurke-Ernzerhof generalized gradient approximation [37]. This approximation yields a semimetallic character but accurately describes the valence bands. More advanced first-principles calculations do not significantly modify the valence band dispersion [20].

Tellurium is a small-gap semiconductor, heavily p-doped due to vacancies. The valence band is formed by $p$ orbitals, and consists of two manifolds. Each manifold contains six connected bands [20,38] and forms an elementary band representation ${ }^{1} \bar{E}^{2} \bar{E} \uparrow G(6)$ [39]. In Fig. 2, we focus on the dispersion of the topmost elementary band representation arising from the Te lone pairs electrons $[19,40]$. Figure 2(a) shows the bulk BZ, and the colors highlight the highsymmetry planes parallel to the cleavage surface. Here, we focus on the Fermi surface, which is shown in Fig. 2(b) for three selected values of the perpendicular wave vector $k_{z}$. It consists of tiny hole pockets, which are better visible in the insets showing a magnified view at the $\mathrm{H}$ point for the three photon energies $[19,23,24]$. The pattern of the pockets evolves from a square $(60 \mathrm{eV})$ to a rectangle $(90 \mathrm{eV})$ and back to a square $(120 \mathrm{eV})$, according to the periodicity of the bulk BZ. Additional information about the 3D band 

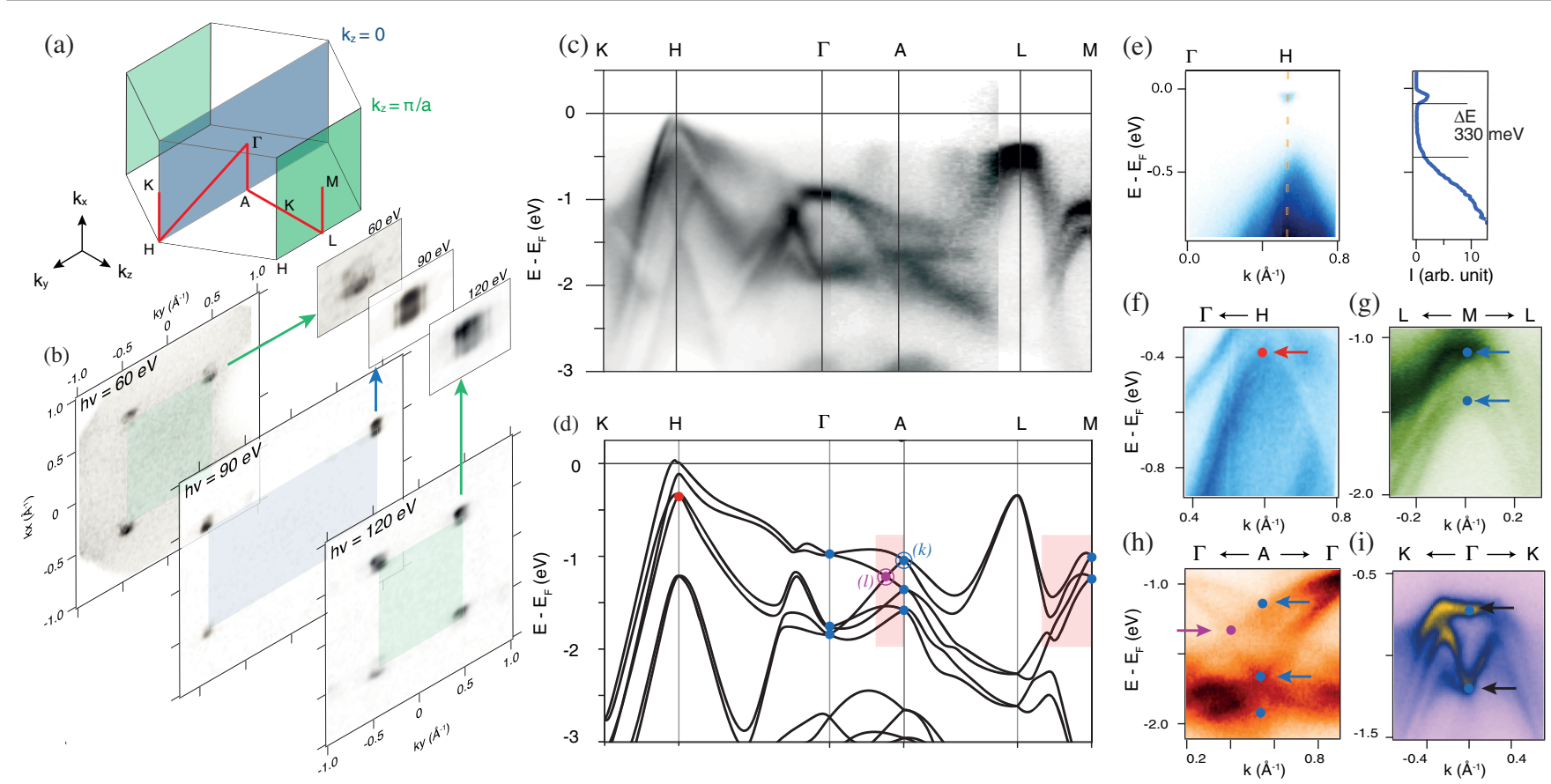

FIG. 2. Band structure and topological classification. (a) Te bulk BZ. Colors indicate the planes parallel to the surface termination for $k_{z}=0$ (blue) and $k_{z}=\pi / a$ (green), where $k_{z}$ is the direction orthogonal to the surface. (b) Fermi surface measured at different photon energies $(60 \mathrm{eV}, 90 \mathrm{eV}$, and $120 \mathrm{eV}$, respectively). Insets show a magnified view on the pocket at the $\mathrm{H}$ point. (c) Band dispersion along the high-symmetry direction indicated by red lines in (a), measured by varying the photon energy between 60 and $120 \mathrm{eV}$. (d) Calculated band structure along the same directions. Colored dots indicate the position of the Weyl nodes experimentally resolved in panels (f)-(i). Red rectangles highlight the regions in which spin polarization is shown in Fig. 3. We indicate with $(k)$ and $(l)$, respectively, the KW and the accordion Weyl points for which the spin texture is presented in Fig. 3. (e) (left) Band dispersion around the $\mathrm{H}$ point, measured at $19 \mathrm{eV}$ photon energy, upon alkali doping. The bottom of the conduction band is shifted below $E_{F}$. (right) Energy distribution curve at the H point. (f)-(i) Magnified view of the band dispersion where different Weyl fermions are resolved. They are protected by $T$ symmetry, by the $C_{3}$ screw rotational axis, or by their combination. Data have been acquired at photon energies equal to $19 \mathrm{eV}$ (f), $60 \mathrm{eV}(\mathrm{g})$, and $90 \mathrm{eV}(\mathrm{h})$ and (i), respectively.

dispersion is given in the Supplemental Material [41]. Figures 2(c) and 2(d) compares the experimental and theoretical dispersion along the cuts indicated by red lines in Fig. 2(a). Alkali metal (K) deposition lowers the minimum of the conduction band below the Fermi level $\left(E_{F}\right)$, and reveals a $\sim 330 \mathrm{meV}$ band gap at the $\mathrm{H}$ point, measured as the distance between the two spectral leading edges, as shown in Fig. 2(e).

As a result of broken $P$ symmetry and large atomic spin orbit coupling, all bands are spin polarized and split in energy. Several band crossings can be identified in our data. These Weyl nodes have been classified according to the mechanism protecting the degeneracy [18-22]. In chiral crystals, $T$ symmetry alone stabilizes KramersWeyl (KW) fermions [21]. In Figs. 2(g)-2(i), we resolve several of these Weyl nodes, indicated by blue dots and shown for clarity also in the calculations of Fig. 2(d). KW fermions are nodes protected by the Kramers' spin degeneracy, and therefore they are pinned at TRIM points and, in contrast to conventional Weyl fermions, they cannot annihilate as the result of small distortions of the lattice.
Among the Weyl nodes observed at the A point [Fig. 2(h)], the middle one is a composite Weyl fermion with a chiral charge equal to 3 [20] due to the combined action of $T$ and $C_{3}$ symmetries. Composite fermions correspond to two or multiple Weyl points with a monopole chiral charge brought together onto a highsymmetry point by the action of a rotational symmetry. As a result, composite Weyl fermions exhibit chiral charges larger than 1 [43]. In order to respect the connectivity of the bands between the $\Gamma$ and A points imposed by the compatibility relations of the irreducible representations, the valence bands must form additional accordion Weyl points. This term refers to the $n-1$ necessary crossings of $2 n$ bands that are connected along a high-symmetry direction and are doubly degenerate at its ends [22]. In Figs. 2(h) and 2(d), we indicate with a violet dot the accordion node for which the spin texture is presented in Fig. 3. Finally, although $\mathrm{H}$ is not a TRIM point, additional Weyl points, enforced there by the $C_{3_{1}}$ axis, were reported by a previous ARPES investigation [23]. We indicate one such Weyl point with a red dot in Figs. 2(f) and 2(d). 
(a)

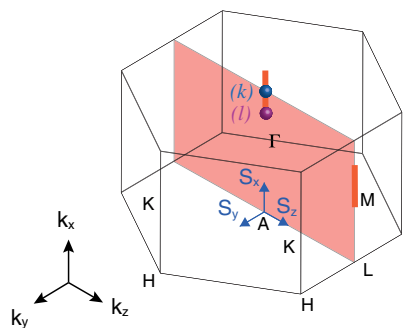

(b)

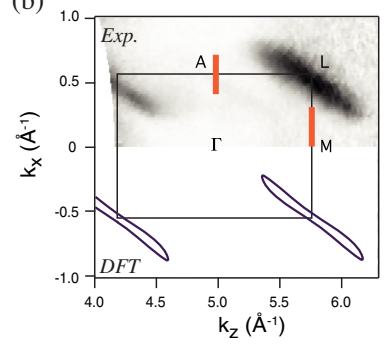

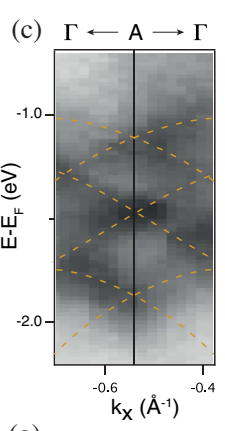

(g) L

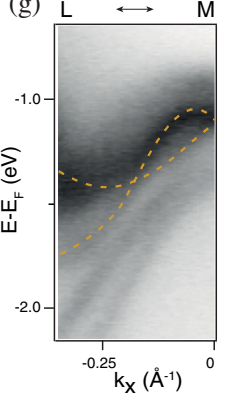

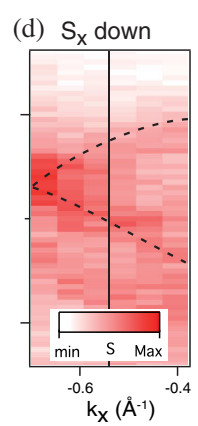
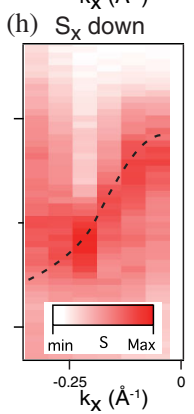
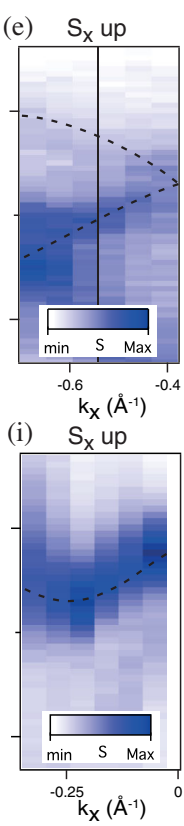

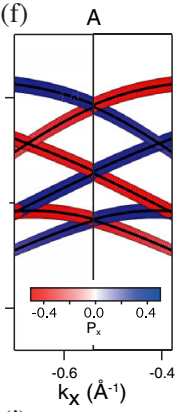

(j)

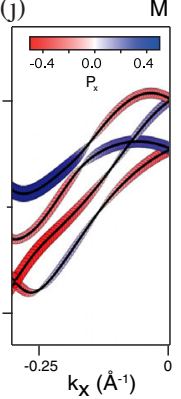

(k) $\mathrm{S}_{\mathrm{x}}$

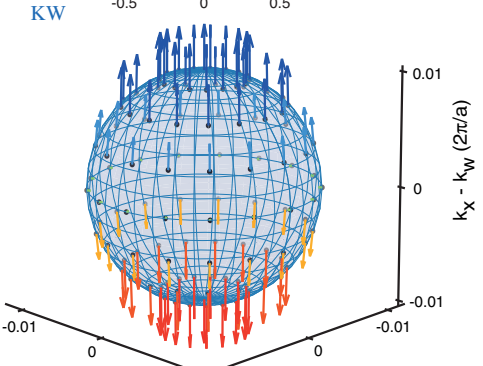

(1) $k_{z}-k_{w}(2 \pi / a) 0.01 \quad 0.01 \quad k_{y}-k_{w}(2 \pi / a)$

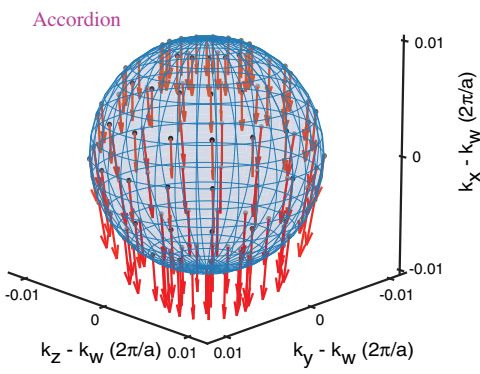

FIG. 3. Spin texture of the Weyl points. (a) The $k_{y}=0$ plane (red) where we focus our spin-resolved study. (b) Constant energy map in this plane $0.5 \mathrm{eV}$ below $E_{F}$ : (upper half) measured by varying the photon energy between 60 and $140 \mathrm{eV}$; (lower half) density functional theory calculations. Red lines indicate where the spin polarization has been measured, corresponding to red rectangles of Fig. 2(d). (c)(e) Spin-integrated and spin-resolved band dispersion, measured at $38 \mathrm{eV}$ photon energy, around the A point, for the radial component of the spin vector, parallel to $k_{x}$. (f) Calculated spin polarization for the radial component along the $\Gamma$ A direction. (g)-(j) Same as in panels (c)-(f) but for the band dispersion measured at $60 \mathrm{eV}$ along the ML direction. (k) and (l) show the orientation of the spin vector field calculated on a sphere of radius $0.01(2 \pi / a)$ centered at the KW point (k) and at the accordion Weyl point (l), as schematized in panel (a). The color scale indicates the magnitude of the $S_{x}$ component.

Theory also predicts at the bottom of the conduction band a Weyl point $[12,19,24]$ that is responsible for the sign change of the circular photogalvanic effect measured as a function of temperature [5,12]. Although it is not resolved in the data of Fig. 2(e), our results show that the bottom of the conduction band and this Weyl point can easily be tuned across $E_{F}$ by doping. In the present work, this is achieved by adsorption of alkali metal on the surface, but a similar doping could also be realized in the bulk. Even more interestingly, the position of the Weyl point could be tuned at $E_{F}$ by external gating of a thin exfoliated flake [17], thus paving the way to the study of anomalies in the magnetotransport properties of Te [44].

The spin properties of these exotic fermions, namely their peculiar radial spin texture [21], have not yet been measured. In Fig. 3, we provide an experimental confirmation of this unique property. We focus our attention on the $\Gamma A$ and ML high-symmetry directions within the $k_{y}=0$ plane highlighted in the bulk BZ of Fig. 3(a). Figure 3(b) shows a constant energy map taken in this plane $0.5 \mathrm{eV}$ below $E_{F}$, comparing experiment (upper part) and theory (bottom part). At this energy, the valence band forms an ellipsoid centered at the $\mathrm{L}$ points, the corners of the rectangle formed by the intersection of the $\mathrm{BZ}$, and the $k_{y}=0$ plane. Notice, however, that the contours do not exhibit $M$ symmetries with respect to the $\Gamma A$ and
ML high-symmetry directions. This is, to the best of our knowledge, the first report of such a remarkable lack of $M$ symmetry in the bulk electronic structure, a direct consequence of the crystal's chirality.

The reference frame for the spin polarization is indicated by blue arrows in Fig. 3(a). Along the ГA line connecting two opposite KW points, the $S_{x}$-hereafter radial-component is parallel to the wave vector of the electron. The two remaining components, $S_{y}$ and $S_{z}$, are respectively parallel and perpendicular to the sample surface. Figure 3(c) shows the spin-integrated band dispersion around the A point. The spin-down and spin-up projections of the radial component $S_{x}$, Figs. 3(d) and 3(e), show clear differences. For each spin projection, only half of the bands are visible, indicated by dashed lines. The radial component of the spin-polarized band structure is well reproduced by the $a b$ initio calculations of Fig. 3(f). The fully vectorial information of the spin texture is discussed in the Supplemental Material [41], where we show that the $S_{y}$ and $S_{z}$ projections are zero within our experimental sensitivity.

We further address the spin polarization around the $\mathrm{M}$ point in Figs. 3(g)-3(j). Also in this case, the spin-up [Fig. 3(i)] and spin-down [Fig. 3(h)] projections show different band dispersions and are well reproduced by the calculations [Fig. 3(j)]. The radial component of the spin 
polarization is the dominant term. Nevertheless, along this direction the out-of-plane components are not negligible (see Supplemental Material [41]). This observation, which is accounted for by our ab initio calculations, reflects the different symmetry of the two directions. While the simultaneous presence of the $C_{3_{1}}$ and the $C_{2}$ axes along $\Gamma$ A imposes $S_{y}$ and $S_{z}$ to be null, ML exhibits a $C_{2}$ symmetry that cancels only the $S_{y}$ component.

It is important to realize that the radial spin texture is enforced by symmetry only along high-symmetry directions and that its evolution in the 3D BZ is more complex. Figure 3(k) displays the spin vector field on a small sphere of radius $0.01(2 \pi / a)$ centered at the $\mathrm{KW}$ point at $\mathrm{A}$, as in Fig. 3(a). The spin direction flips from small and inward at the equator to large and outward at the poles. By contrast, Fig. 3(1) shows the spin polarization around the accordion Weyl point, where no symmetries enforce the radial spin texture. In the Supplemental Material [41], we discuss in more detail the spin projection on different planes cutting through the spheres [41].

Symmetry is the fundamental ingredient to control the radial spin configuration, which is indeed a general property of chiral crystals and not only of KW points. It is also enforced along a threefold axis where additional twofold rotational symmetries are present. In Te, these conditions are satisfied also at the $\mathrm{K}$ and $\mathrm{H}$ points. The Fermi surface, which is centered at the latter, is therefore fully spin polarized with a radial spin texture, as shown in the Supplemental Material [41]. The calculation of Fig. 3(k) shows that the 3D spin texture is more complex than the hedgehog configuration expected for a magnetic monopole [45]. A full classification of the spin vector field geometry is beyond the scope of this study, and it will be the subject of a future investigation.

In summary, we have investigated the spin and electronic properties of tellurium, one of the simplest realizations of a chiral crystal. We experimentally confirm the existence of several exotic fermions: Kramers-Weyl, composite, and accordionlike Weyl nodes. We also prove that their position can be tuned at the Fermi level by surface doping. The breaking of inversion and mirror symmetries enforces a unique spin texture that is radial along the directions connecting high-symmetry points $\Gamma, \mathrm{A}, \mathrm{K}$, and $\mathrm{H}$. We thus report the first experimental evidence of the radial spin polarization of Kramers-Weyl fermions. Our experimental findings not only provide new microscopic insights into the response of Te to external magnetic fields but also demonstrate that spin can arrange in momentum space with a vectorial field more complex than the so-farconsidered hedgehog configuration. The analogy with the alignment taken by local magnetic moments in real space calls for a detailed classification of the spin textures that can be realized in chiral materials.

We acknowledge financial support by the Swiss National Science Foundation (SNSF), in particular L. T. acknowledges support under Grant No. 200020_188648, M. F. under Grant No. P2ELP2_181877, and S. M. under Grant No. P300P2-171221. D. G. M., S. S. T., and O. V. Y. acknowledge the support by the NCCR. S. S. T. acknowledges support from the European Union Horizon 2020 Research and Innovation Program (ERC-StG-Neupert757867-PARATOP) and Swiss National Science Foundation (Grant No. PP00P2_176877). M. P., S.P., and M.C. acknowledge the support by the ERC Advanced Grant No. 695197 (DYNAMOX)) and the Swiss National Science Foundation NCCR:MUST Grant. We gratefully acknowledge support from the Department of Energy, Office of Science under Grant No. DE-FG0207ER46405. J. W. acknowledges a National Science Foundation Graduate Research Fellowship under Grant No. 1144469. All first-principles calculations were performed at the Swiss National Supercomputing Centre (CSCS) under Projects No. s832 and No. s1008. We acknowledge Elettra Sincrotrone Trieste for providing access to its synchrotron radiation facilities. This work has been partly performed in the framework of the nanoscience foundry and fine analysis (NFFA-MIUR Italy Progetti Internazionali) facility. This research used resources of the Advanced Light Source, which is a DOE Office of Science User Facility, under Contract No. DE-AC0205CH11231.

* Corresponding author. alberto.crepaldi@epfl.ch

[1] P. W. Bridgman, Proc. Natl. Acad. Sci. U.S.A. 10, 411 (1924).

[2] K. von Klitzing and G. Landwehr, Phys. Status Solidi B 45, K119 (1971).

[3] T. Englert, K. von Klitzing, R. Silbermann, and G. Landwehr, Phys. Status Solidi B 81, 119 (1977).

[4] V. M. Asnin, A. A. Bakun, A. M. Danishevskii, E. L. Ivchenko, G. E. Pikus, and A. A. Rogachev, JETP Lett. 28, 74 (1978), http://www.jetpletters.ac.ru/ps/1557/ article_23830.shtml.

[5] V. M. Asnin, A. A. Bakun, A. M. Danishevskii, G. E. Pikus, and A. A. Rogachev, Solid State Commun. 30, 565 (1979).

[6] G. L. J. A. Rikken and N. Avarvari, Phys. Rev. B 99, 245153 (2019).

[7] T. Furukawa, Y. Shimokawa, K. Kobayashi, and T. Itou, Nat. Commun. 8, 954 (2017).

[8] L. E. Vorob'ev, E. L. Ivchenko, G. E. Pikus, I. I. Farbshtein, V. Shalygin, and A. V. Shturbin, JETP Lett. 29, 441 (1979), http://www.jetpletters.ac.ru/ps/1454/article_22128.shtml.

[9] V. A. Shalygin, A. N. Sofronov, L. E. Vorobev, and I. I. Farbshtein, Phys. Solid State 54, 2362 (2012).

[10] T. Yoda, T. Yokoyama, and S. Murakami, Sci. Rep. 5, 12024 (2015).

[11] W.-Y. He, X. Y. Xu, and K. T. Law, arXiv:1905.12575v2.

[12] S. S. Tsirkin, P. A. Puente, and I. Souza, Phys. Rev. B 97, 035158 (2018). 
[13] F. de Juan, A. G. Grushin, T. Morimoto, and J. E. Moore, Nat. Commun. 8, 15995 (2016).

[14] C. Şahin, J. Rou, J. Ma, and D. A. Pesin, Phys. Rev. B 97, 205206 (2018).

[15] S. Lin, W. Li, Z. Chen, J. Shen, B. Ge, and Y. Pei, Nat. Commun. 7, 10287 (2015).

[16] H. Peng, N. Kioussis, and G. J. Snyder, Phys. Rev. B 89, 195206 (2014).

[17] Y. Wang, G. Qiu, R. Wang, S. Huang, Q. Wang, Y. Liu, Y. Du, W. A. Goddard III, M. J. Kim, X. Xu et al., Nat. Electron. 1, 228 (2018).

[18] J. L. Mañes, Phys. Rev. B 85, 155118 (2012).

[19] M. Hirayama, R. Okugawa, S. Ishibashi, S. Murakami, and T. Miyake, Phys. Rev. Lett. 114, 206401 (2015).

[20] S. S. Tsirkin, I. Souza, and D. Vanderbilt, Phys. Rev. B 96, 045102 (2017).

[21] G. Chang, B. J. Wieder, F. Schindler, D. S. Sanchez, I. Belopolski, S. M. Huang, B. Singh, D. Wu, T.-R. Chang, T. N. S.-Y. Xu et al., Nat. Mater. 17, 978 (2018).

[22] Y.-H. Chan, B. Kilic, M. M. Hirschmann, C.-K. Chiu, L. M. Schoop, D. G. Joshi, and A. P. Schnyder, Phys. Rev. Mater. 3, 124204 (2019).

[23] K. Nakayama, M. Kuno, K. Yamauchi, S. Souma, K. Sugawara, T. Oguchi, T. Sato, and T. Takahashi, Phys. Rev. B 95, 125204 (2017).

[24] L. A. Agapito, N. Kioussis, W. A. Goddard, and N. P. Ong, Phys. Rev. Lett. 110, 176401 (2013).

[25] T. Ideue, M. Hirayama, H. Taiko, T. Takahashi, M. Murase, T. Miyake, S. Murakami, T. Sasagawa, and Y. Iwasa, Proc. Natl. Acad. Sci. U.S.A. 116, 25530 (2019).

[26] D. Rodriguez, A. A. Tsirlin, T. Biesner, T. Ueno, T. Takahashi, K. Kobayashi, M. Dressel, and E. Uykur, Phys. Rev. Lett. 124, 136402 (2020).

[27] J. H. Dil, J. Phys. Condens. Matter 21, 403001 (2009).

[28] M. Z. Hasan and C. L. Kane, Rev. Mod. Phys. 82, 3045 (2010).

[29] G. Landolt, S. V. Eremeev, Y. M. Koroteev, B. Slomski, S. Muff, T. Neupert, M. Kobayashi, V. N. Strocov, T.
Schmitt, Z. S. Aliev et al., Phys. Rev. Lett. 109, 116403 (2012).

[30] M. Sakano, M. Hirayama, T. Takahashi, S. Akebi, M. Nakayama, K. Kuroda, K. Taguchi, T. Yoshikawa, K. Miyamoto, T. Okuda et al., Phys. Rev. Lett. 124, 136404 (2020).

[31] A. Bradley, Philos. Mag. 48, 477 (1924).

[32] S. Andersson, D. Andersson, and I. Marklund, Surf. Sci. 12, 284 (1968).

[33] A. Nussbaum, Proc. IRE 50, 1762 (1962).

[34] L. Petaccia, P. Vilmercati, S. Gorovikov, M. Barnaba, A. Bianco, D. Cocco, C. Masciovecchio, and A. Goldoni, Nucl. Instrum. Methods Phys. Res., Sect. A 606, 780 (2009).

[35] C. Bigi et al., J. Synchrotron Radiat. 24, 750 (2017).

[36] P. Giannozzi, S. Baroni, N. Bonini, M. Calandra, R. Car, C. Cavazzoni, D. Ceresoli, G. L. Chiarotti, M. Cococcioni, I. Dabo et al., J. Phys. Condens. Matter 21, 395502 (2009).

[37] J. P. Perdew, K. Burke, and M. Ernzerhof, Phys. Rev. Lett. 77, 3865 (1996).

[38] L. Michel and J. Zak, Phys. Rev. B 59, 5998 (1999).

[39] L. Elcoro, B. Bradlyn, Z. Wang, M. G. Vergniory, J. Cano, C. Felser, B. A. Bernevig, D. Orobengos, G. de la Flor, and M. I. Aroyo, J. Appl. Crystallogr. 50, 1457 (2017).

[40] J. D. Joannopoulos, M. Schlüter, and M. L. Cohen, Phys. Rev. B 11, 2186 (1975).

[41] See Supplemental Material at http://link.aps.org/ supplemental/10.1103/PhysRevLett.125.216402 for additional information about the bulk dispersion, the full vectorial spin components, and the spin structure at the $\mathrm{H}$ point, which includes Refs. [19,30,35,42].

[42] B. Feng, Y.-H. Chan, Y. Feng, R.-Y. Liu, M.-Y. Chou, K. Kuroda, K. Yaji, A. Harasawa, P. Moras, A. Barinov et al., Phys. Rev. B 94, 195134 (2016).

[43] C. Fang, M. J. Gilbert, X. Dai, and B. A. Bernevig, Phys. Rev. Lett. 108, 266802 (2012).

[44] G. Qiu, C. Niu, Y. Wang, M. Si, Z. Zhang, W. Wu, and P. D. Ye, Nat. Nanotechnol. 15, 585 (2020).

[45] J. H. Dil, Physics 13, 45 (2020). 